\title{
Q\&A: Brian Greene on music and string theory
}

Brian Greene, author of best-selling books The Elegant Universe and The Fabric of the Cosmos, is a theoretical physicist at Columbia University, New York. As an orchestral work based on his 2008 children's book, Icarus at the Edge of Time, premieres next week, Greene discusses black holes and how music might portray the physics of warped space-time.

\begin{abstract}
How have you reworked the Greek myth of Icarus?

Instead of donning wax wings and flying to the Sun, in this story a boy builds his own spaceship and, against his father's warning, flies close to a black hole. Black holes have a reputation for reaching out and sucking things in, but they're not like vacuum cleaners - if you stay outside the black hole's boundary, or event horizon, you can pull away. The boy does just that, but as Albert Einstein taught us, black holes affect one's passage through time. When the boy returns from his journey, it is 10,000 years later. The life he knew is gone. His father is gone.
\end{abstract}

\section{Isn't that rather grim for a children's story?}

It is dark from one perspective but brighter from another. At first I didn't want to force the book on my five year old. Only recently did my wife read it to him, and he cried at the end. Since then, he asks me questions almost daily, such as if the black hole was smaller, might less time have passed so the father would still be alive? Time dilation and general relativity now matter to my five year old. That's unusual, and shows what such an undertaking can do. It can make science really matter.

\section{Why were you attracted to the myth?}

It struck me as odd, troublesome even; the original Icarus pays for his courage with his life, but that is not the way things should work. As scientists, we know that you must push the boundaries. You go into the darkness, not knowing how it will turn out, but being willing to grapple with the consequences. We have seen that during the atomic age and, more recently, with the human genome, where we are still struggling to come to terms with our new-found power.

\section{How is your story adapted to the stage?} It has a narrator and orchestra, and a film is projected behind it. Philip Glass composed the score. David Henry Hwang, who wrote the 1988 play M. Butterfly, helped me to adapt the text. The visual artists $\mathrm{Al}$ and $\mathrm{Al}$ are creating the film, which attempts in an impressionistic way to suggest what it is like to travel near the edge of a black hole.

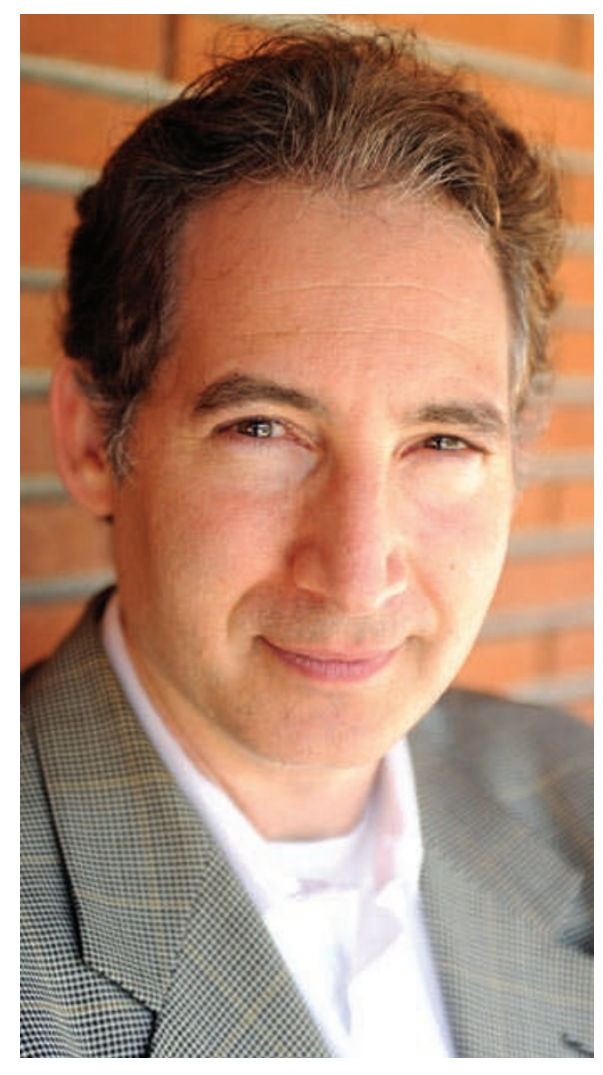

What was it like to work with Philip Glass, the composer of operas about physicists Johannes Kepler and Einstein?

His interest in science goes deep. When The Elegant Universe came out in 1999, he called me to discuss some of the chapters. After he agreed to compose the music for Icarus at the Edge of Time, I would explain the physics of warped space and time, and he would struggle to represent it musically. For instance, as the boy got near the black hole, would you want two lines of music playing against each other, one for his time frame and one for the rest of the Universe? Should you represent gravitational redshift with changes in frequency? He has tried, in an artful and poetic way, to capture some of the physics.

\section{Meanwhile, what of your research?} Many string theorists are asking whether the evolution of the Universe over time could give us an insight into how likely our Universe is to exist among many possible universes. At Columbia we are studying 'quantum tunnellings', which may punch through a barrier from one universe to another. We're studying the ways that quantum mechanics can generate multiple universes. I'm writing a popular book about these, which asks the essential question: when we talk about universes beyond our own, is it science?

\section{Has string theory helped us to understand black holes?}

String theory has given us insights into black holes that could not have been obtained solely through Einstein's theory of general relativity. In 1974, Stephen Hawking asserted that black holes are not completely black; they radiate quantum mechanically, which means they have entropy, a measure of disorder. For more than 20 years, no one could figure out where the disorder was stored. Then in 1996, physicists Andrew Strominger and Cumrun Vafa at Harvard University resolved that problem using string theory. It was a profound discovery. The fact that string theory could articulate something so fundamental convinced many of us that it had the capacity to describe physics as we know it.

\section{What do you make of those who are sceptical of string theory?}

String theory is an attempt to study some of the deepest questions that we have ever studied scientifically. All of us agree that the theory has yet to be established experimentally. We're still trying to understand it well enough to make contact with experiment. That we haven't yet reached that point is not a failure of the theory. It's a challenge we're trying to rise to.

Jascha Hoffman is a journalist based in San Francisco, California.

e-mail: jascha@jaschahoffman.com

\section{Icarus at the Edge of Time}

Narration adapted by Brian Greene and

David Henry Hwang. Music by Philip Glass.

Film created and directed by Al and Al.

At the World Science Festival:

6 June, NYU Skirball Center, New York

At the Royal Society's See Further festival: 3-4 July, Royal Festival Hall, London 